\title{
A Flexible Approach for Smart Management of Transmissions in Power Line Communications
}

\author{
Mario Collotta and Salvatore Tirrito \\ Facoltà di Ingegneria e Architettura, Università degli Studi di Enna-Kore, Cittadella Universitaria, 94100 Enna, Italy \\ Correspondence should be addressed to Mario Collotta; mario.collotta@unikore.it
}

Received 25 June 2014; Revised 2 November 2014; Accepted 12 November 2014; Published 8 December 2014

Academic Editor: Lixin Gao

Copyright (C) 2014 M. Collotta and S. Tirrito. This is an open access article distributed under the Creative Commons Attribution License, which permits unrestricted use, distribution, and reproduction in any medium, provided the original work is properly cited.

\begin{abstract}
Power line communications (PLCs) refer to a technology based on the existing electrical wiring to transmit data among the devices connected to the network. The PLC technology is an excellent solution widely studied and analysed by researchers, even in those areas characterized by strict requirements, such as industries. In this paper, a technique based on fuzzy logic, for the dynamic management of the amplitude of the signal emitted by the devices of a power line network, is proposed. The main aim is to manage the amplitude of the transmission signal in order to reduce the noise introduced into the network, and, as a consequence, the power consumption, increasing data transmission quality of network in terms of Quality of Service (QoS). This solution has been implemented into embedded systems based on the ADD1010, a power line System on Chip (SoC), and tested through a real scenario realized in laboratory.
\end{abstract}

\section{Introduction}

Recently, the power grid has become increasingly used also to allow the access to private data and exchange information among devices. This introduces the concept of power line communications (PLCs) [1]. This approach provides data transmissions using frequencies higher (a few tens $\mathrm{kHz}$ ) than $50 \mathrm{~Hz}$ (or $60 \mathrm{~Hz}$ ) related to the power supply. The PLCs are hampered by several factors: attenuation at the frequencies of interest [2, 3]; noises [3-6]; interference due to electronic devices connected to the system [7]; crosscoupling interferences [8]. However, the PLC technology is characterized by several strengths [9]: it does not need additional wires for data transmission and allows maintaining the connections even in case of through walls and curvatures. An aspect that must be considered concerns the management of the amplitude of the transmission signal in order to reduce the noise introduced into the network and, at the same time, the power consumption produced by the devices. Moreover, the noise measured in a communication medium leads to a proportional reduction of the network Throughput/Workload (TH/WL), that is, the ratio between the number of packets received and number of packets sent, that can be managed by dynamically varying the amplitude of the signal emitted by each device. Taking into account the network information, such as the master-slave distance (link length) and the link quality (noise level measured in the communication medium), it is possible to reduce the power consumption of each device and, then, the noise introduced into the network by means of a fuzzy logic controller. The fuzzy logic controller will evaluate, before each transmission, the distance from each slave and the disturbance (link quality) measured on the transmission channel due to the network load. Several measures have been carried out through a real test-bed scenario. In more detail, we measured the active power consumption, the Throughput/Workload ratio of the network, and the noise level introduced into the network, in terms of cross-coupling interference. The obtained results are very promising and show that the road to follow to ensure the optimal management of the amplitude of the emitted signal is correct and must be pursued. The paper is organized as follows: Section 2 deals with the main literature related works; Section 3 describes the proposed approach while Section 4 describes the real test-bed implemented showing, at the same time, the obtained results. Finally, Section 5 summarizes the paper reporting the conclusions. 


\section{Related Works}

In literature, little attention has been given to power consumption related to power line communications. In our opinion, a careful study focused on some parameters related to power consumption of devices should be conducted in order to reduce the total power consumption of the network and the noise introduced by the same devices. In industrial environments there are hundreds (thousands) of nodes. As a consequence, energy savings would result in an important benefit for the management of the plant itself. During our studies, we noticed that, in literature, the power line communications have been widely investigated in several aspects.

In [10], the authors analyse the effects of the impulsive noise in narrowband PLC systems and derive an explicit formula of bit error rate (BER) and its upper bound for an uncoded and coded orthogonal frequency division multiplexing (OFDM) schemes in PRIME [11]. Their analyses and the upper bound are then validated through simulations. Furthermore, the authors propose an efficient time domain interleaver (TDI) technique to improve the performance of PRIME by spreading the effect of impulsive bursts over a large number of OFDM symbols. Many other works, in literature, concern the study of techniques in order to reduce the noise in power line networks. In [12], the authors propose clipping scheme and equalizer as methods to reduce the effects of impulsive noise and channel attenuation in power line communication systems. The clipping is a mechanism through which it is possible to cut off the amplitude of the received signal over a threshold value, without its phase change, in order to reduce noise effects. The equalizer compensates for effects of PLC channel. The performance is evaluated in terms of bit error rate (BER). From simulation results, the authors confirm that their proposed clipping scheme has slightly better performance than conventional PLC system. Moreover, the authors claim that the results of their paper can be applied to PLC systems for smart grid. In [13], the authors propose an optimal Clipping/Blanking nonlinearity technique for impulsive noise reduction in narrowband $(9 \mathrm{kHz}-490 \mathrm{kHz})$ PLC system. The authors use the Clipping/Blanking memoryless nonlinearity for mitigating the effect of impulsive noise in OFDM systems over narrowband PLC channels. The authors also propose a method to find optimal threshold, corresponding to different values of signal-to-noise ratio (SNR), which minimizes the bit error rate (BER). This proposed method further improves the performance of Clipping/Blanking technique in the presence of impulsive noise over narrowband PLC systems.

These approaches leave out some very important aspects: power consumption and acceptable network performance, mainly in terms of Throughput/Workload. The latter factor is strongly influenced by the disturbance measured on the channel itself. An uncontrolled growth of the noise can determine an increasing packet error rate and therefore the degradation of network performance.

Only a few researchers (i.e., [14, 15]) have analyzed this point of view, but they determine other factors that influenced the requirements of network performance. For example, a novel technique of using single frequency network with

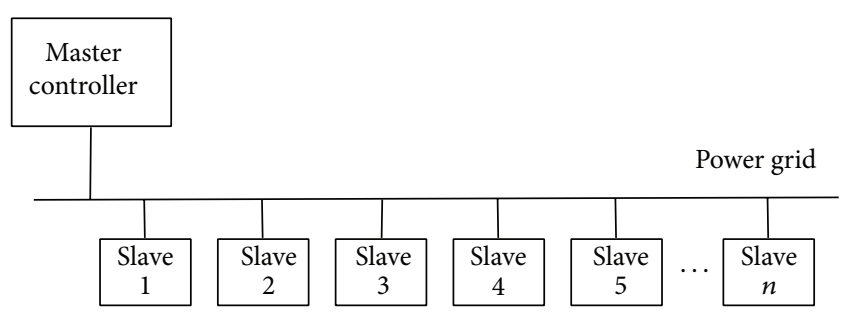

FIgURE 1: Power line network topology.

flooding based routing that converts the channel congestion into channel cooperation for a narrowband PLC application is described in [16]. This technique is supposed to achieve better channel congestion performance by using PLC as compared to the available wireless communication techniques.

What emerged, from this synthesis of the state of the art, is that there are no studies in literature concerning the dynamic management of the signal emitted by devices of a power line network. From this point, the inspiration for the theme is covered in this paper.

\section{The Proposed Approach}

It is always seen that the network performance degrades at peak traffic hours [17]. Quality of Service (QoS) is a serious problem especially in cases where absolute reliability of communication is required such as remote monitoring, and signals from PLC system installed in adjacent flats may crosstalk to other PLC systems and so forth.

A power line network is characterized by a master and several slaves that communicate each other using the power grid, as depicted in Figure 1.

Data throughput could be degraded due to this interference and/or due the increase of workload network. As a consequence channel congestion is considered a problem in communication. In case of channel congestion, wireless technologies fail to deliver when compared with PLC based technologies as suggested in [17].

Our work differs from other literature approaches, because our work has been conducted in terms of dynamically managing the amplitude in order to obtain some data communication improvement and consequently power consumption reduction of devices. Literature works are lacking in this scope. It is an object underlying this paper to provide a power line communication method in which disturbances of power line communication between power line communication devices by interferences or from noise sources can be reduced in a simple and reliable manner in order to increase the communication quality and the communication reliability. The main aim of this work is to realize an embedded solution in order to dynamically manage the amplitude of the signal emitted by the devices, in order to enhance one of the most important parameters of a data transmission system, the Throughput/Workload, and also try to reduce, even if so small power consumption. This solution has been implemented in the master controller device. The master device dynamically determines, through 


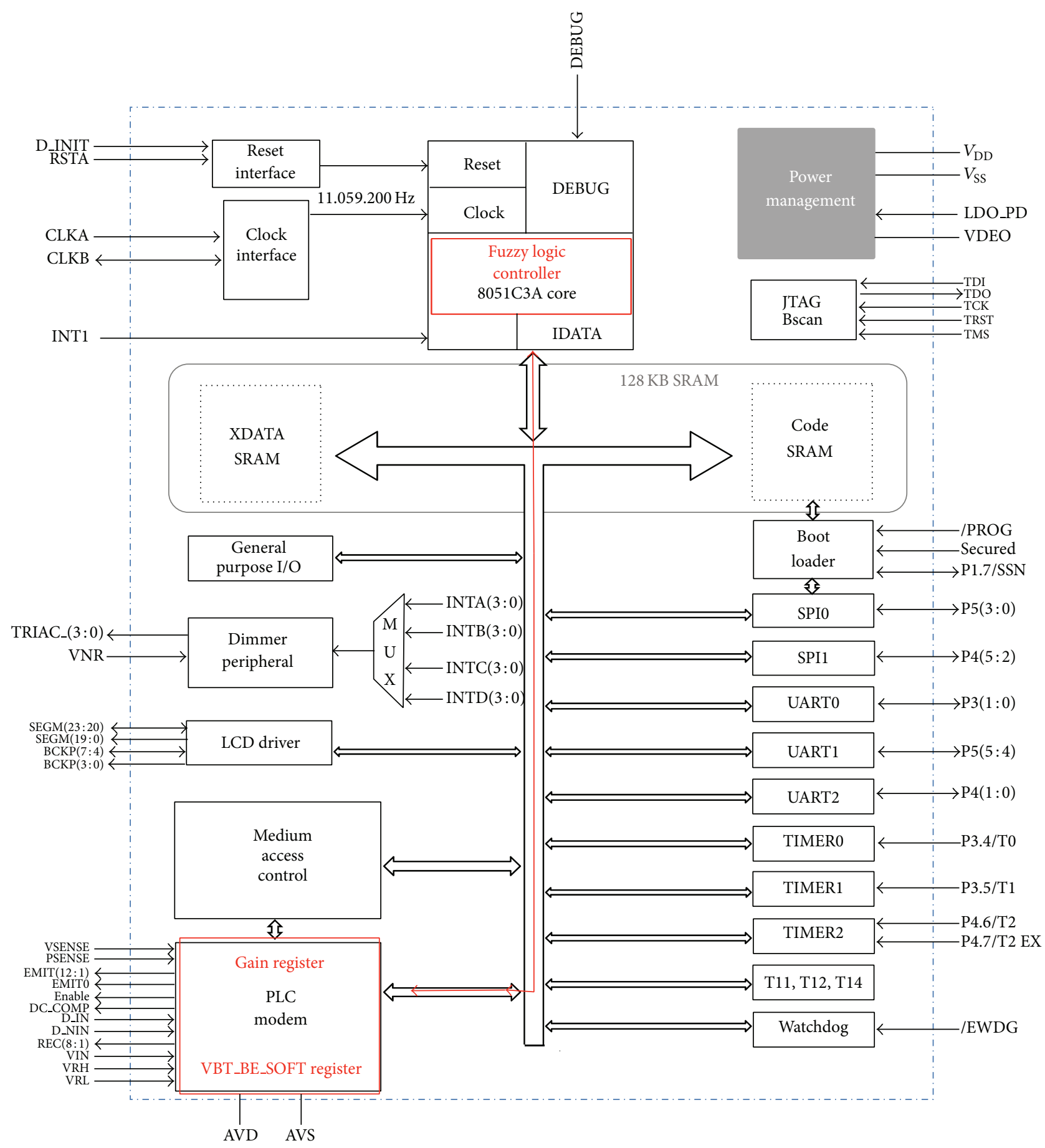

Figure 2: ADD1010 schema.

a fuzzy logic controller, the amplitude of the signal emitted by each slave, based on the master-slave distance and the link quality. Both the master and the slaves are characterized by the ADD1010, a power line communication System on Chip (SoC) that implements a full PLC device using FSK modulation compatible with IEC613434-5-2. It includes an enhanced 8051 microcontroller, a hardwired medium access controller (MAC) and a modem circuit for power line medium [18]. The ADD1010 schema is shown in Figure 2.
As it is possible to see, the fuzzy logic controller has been implemented into the microcontroller (MCU) of the ADD1010 (ADD8051C3A), an enhanced version of the 8051 microcontroller family. The fuzzy logic controller, as explained in detail in Section 3.1, reads a special register (the VTB BE SOFT register of the ADD1010) that contains information related to the link quality in terms of BER and writes on another special register (the Gain Register of the ADD1010) managing in this way the amplitude of the signal 


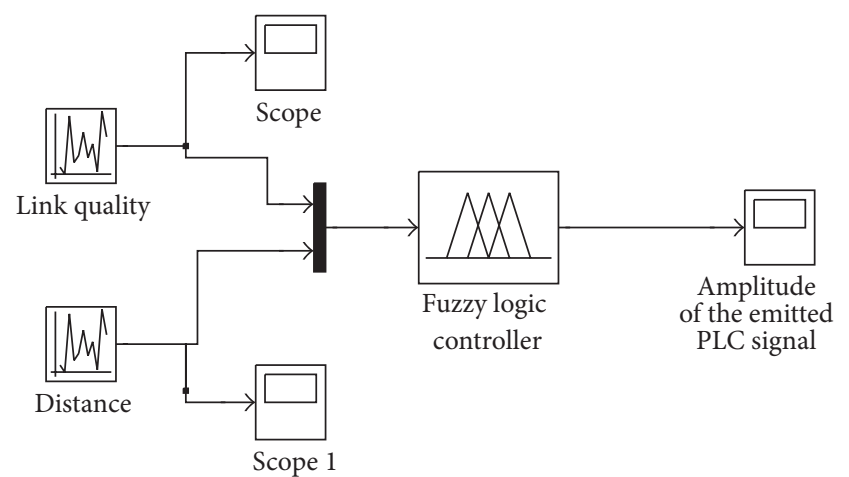

FIgURE 3: The fuzzy system scheme.

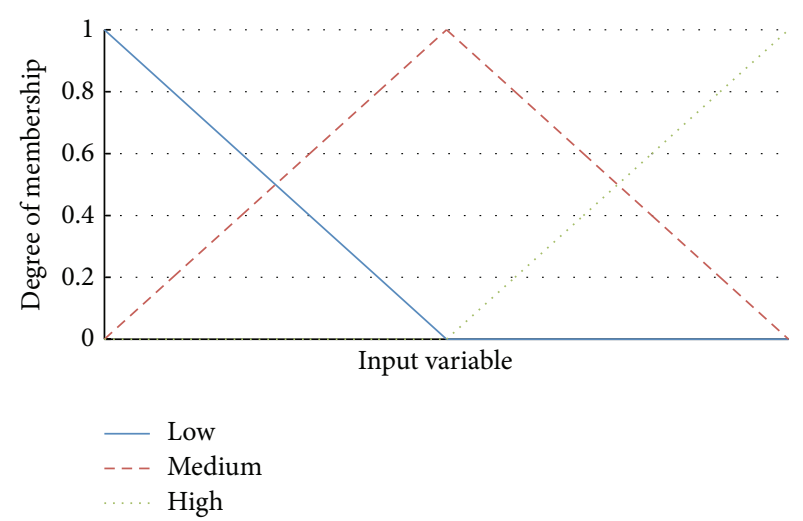

FIGURE 4: Input variables membership functions representation.

membership functions for the output variable (very low, low, medium, high, and very high) have been defined. The input variables have been represented through 3 membership functions because it is always seen that a higher number does not determine an improvement of performance [20]. On the contrary, it would increase the computational hardware implementation complexity [21].

With respect to the output variable, we implemented 5 membership functions. They are suitable to represent with sufficient accuracy the real implemented system. In fact, the membership functions have been developed considering these value ranges:

(i) (input) master-slave distance [0-100] mt;

(ii) (input) link quality [0-7] where 0 represents the lowest quality value while 7 represents the highest;

(iii) (output) amplitude of emitted PLC signal [82-123].

The first input variable, that is, the master-slave distance, has been set at design time. In this way, the master controller knows where each device is placed. The link quality value, instead, can be obtained reading the VTB BE SOFT register of the ADD1010 that stores the number of errors accumulated in a frame reception using the Viterbi algorithm [22]. The amplitude of the emitted PLC signal, that is, the controlled output, is stored in the Gain Register and can assume values from $0 \mathrm{x} 00$ to $0 \mathrm{x} 7 \mathrm{~B}$, that correspond to the range $0-123$ in DEC format.

We have chosen to vary the amplitude of the emitted PLC signal between 82 and 123 because we measured that, for values lower than 82 , the packet loss percentage was too high. Table 1 summarizes the correspondence between the values that can be contained in the Gain Register and their relative Vpp (Volt) values of the sinusoidal modulated FSK signal transmitted into the power grid, upon $50 \mathrm{~Hz}$ with carrier frequency equal to $72 \mathrm{kHz}$ (powering embedded system at 12 VDC).

As previously said, the minimum DEC value that the amplitude can assume is 82 since we verified that lower values determine high percentages of packet loss. Figures 4 and 5 show how all input and output variables, respectively, are generically fuzzyfied. The $Y$-axis specifies the normalized for each input variable (low, medium, and high) and five 


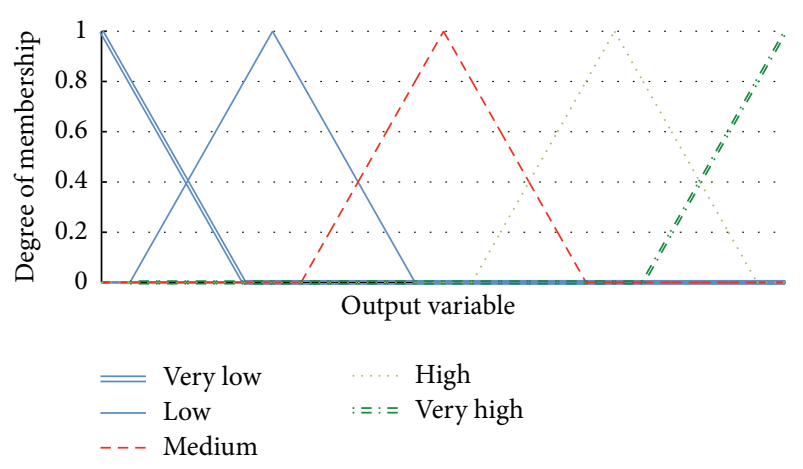

FIgURE 5: Output variable membership functions representation.

TABLE 1: DEC-Vpp valued correspondences.

\begin{tabular}{lcccc}
\hline DEC & Vpp (Volt) & & DEC & Vpp (Volt) \\
\hline 82 & 5.8239 & - & 103 & 6.9469 \\
83 & 5.8641 & - & 104 & 7.0151 \\
84 & 5.9056 & - & 105 & 7.0845 \\
85 & 5.9484 & - & 106 & 7.1553 \\
86 & 5.9925 & - & 107 & 7.2274 \\
87 & 6.0380 & - & 108 & 7.3009 \\
88 & 6.0848 & - & 109 & 7.3757 \\
89 & 6.1330 & - & 110 & 7.4518 \\
90 & 6.1825 & - & 111 & 7.5292 \\
91 & 6.2333 & - & 112 & 7.6080 \\
92 & 6.2854 & - & 113 & 7.6881 \\
93 & 6.3389 & - & 114 & 7.7696 \\
94 & 6.3937 & - & 115 & 7.8524 \\
95 & 6.4499 & - & 116 & 7.9356 \\
96 & 6.5073 & - & 117 & 8.0219 \\
97 & 6.5662 & - & 118 & 8.1087 \\
98 & 6.6263 & - & 119 & 8.1968 \\
99 & 6.6879 & - & 120 & 8.2862 \\
100 & 6.7506 & - & 121 & 8.3770 \\
101 & 6.8147 & - & 122 & 8.4691 \\
102 & 6.8802 & - & 123 & 8.5625 \\
\hline & & & &
\end{tabular}

degree of membership of a variable to the specific membership function; that is, 1 represents the $100 \%$ membership to a specific set. Considering a generic variable, containing a range of values from the minimum to the maximum value that the variable itself can assume, each membership function can be represented by a triangular-shaped membership function. The output value is determined through 9 fuzzy rules (Table 2) based on the IF-THEN statement of classic programming languages and following the classical human reasoning. For example, considering rule 2 , if the link quality is low and the distance is medium, the amplitude of the emitted PLC signal will be high.

The last step is represented by the defuzzification, which finds a single crisp output value from the solution fuzzy space. The defuzzification is performed using the centre-of-gravity method [23].
TABLE 2: Fuzzy rules.

\begin{tabular}{lccc}
\hline Rule & Link quality & Distance & Amplitude \\
\hline 1 & Low & Low & Medium \\
2 & Low & Medium & High \\
3 & Low & High & Very high \\
4 & Medium & Low & Low \\
5 & Medium & Medium & Medium \\
6 & Medium & High & High \\
7 & High & Low & Very low \\
8 & High & Medium & Low \\
9 & High & High & Medium \\
\hline
\end{tabular}

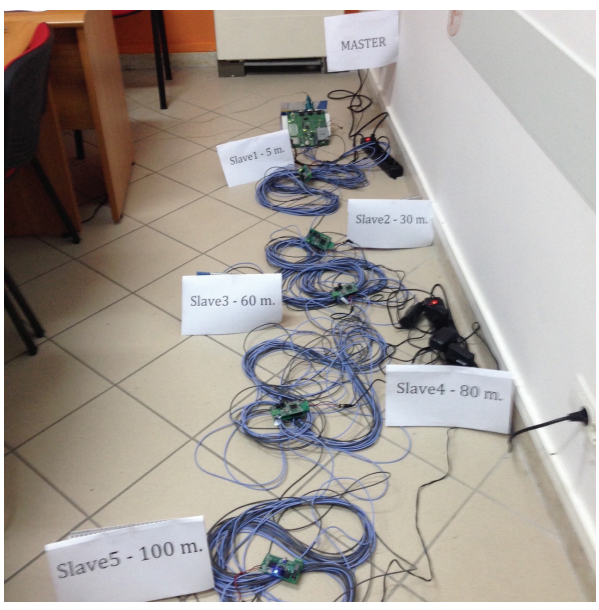

FIGURE 6: Real experimental test-bed.

TABLE 3: Master-slave distances.

\begin{tabular}{lc}
\hline Slave & Distance to master $(\mathrm{m})$ \\
\hline 1 & 5 \\
2 & 30 \\
3 & 60 \\
4 & 80 \\
5 & 100 \\
\hline
\end{tabular}

\section{Performance Evaluation}

In order to verify the validity of the proposed approach, we firstly realized a power line infrastructure that consists of a master and 5 slaves in a bus topology. The slaves have been placed at increasing distance with respect to the master (Table 3) over one phase only.

Figure 6 shows the real test-bed scenario realized in laboratory.

The master, before each transmission to a slave device, determines the amplitude of the emitted signal through the fuzzy controller that processes the link quality level and the master-slave distance. The packet sent by the master contains the amplitude value with which it is transmitting. The slave will respond by transmitting with the same amplitude. We carried out 2000 transmissions with polling frequency equal to 1 second. In Figure 7, it is possible to see the comparison 


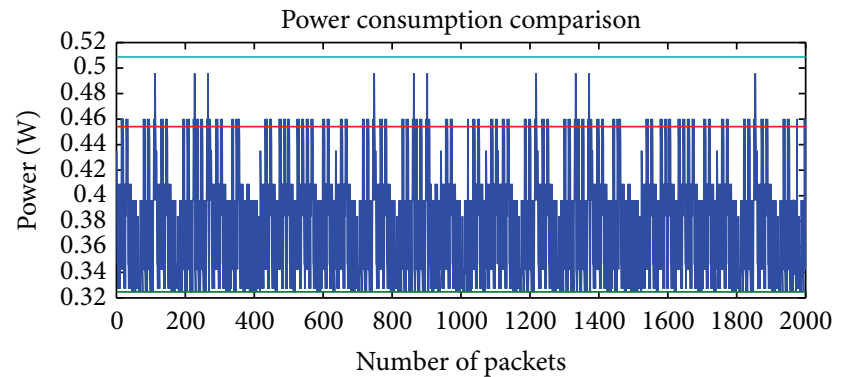

$\begin{array}{ll}\text { - Fuzzy amplitude } & - \text { Amplitude }=111 \\ \text { Amplitude }=82 & \text { Amplitude }=123\end{array}$

FIGURE 7: Power consumption comparison.

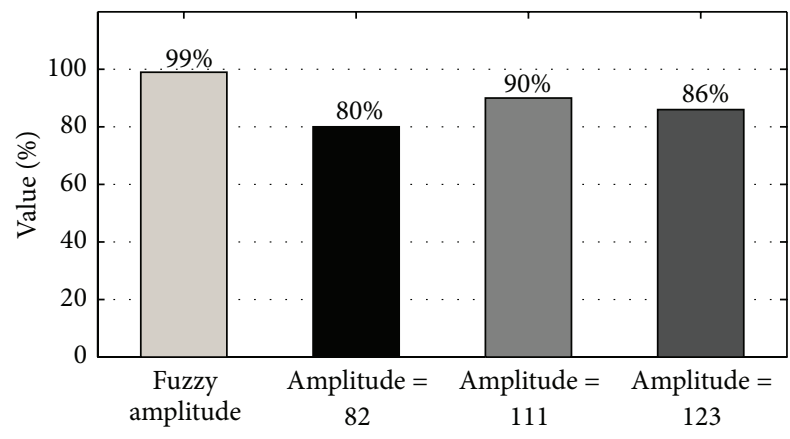

FIGURE 8: Throughput/Workload percentage comparison.

made in terms of power consumption. The power consumption was calculated using the active power formula described in

$$
\langle P\rangle=V_{\mathrm{rms}} * I_{\mathrm{rms}} * \cos \varphi,
$$

where $V_{\text {rms }}$ and $I_{\text {rms }}$ refer to the rms (root mean square) values of voltage and current and $\varphi$ is the phase shift between voltage and current.

The fuzzy logic controller produces power consumption, on average, lower than transmissions done with maximum and medium amplitude (123 and 111 in DEC format, resp.) but higher than transmissions with minimum amplitude (82). This result is however acceptable and reasonable especially considering what gained in terms of TH/WL. In order to measure the TH/WL percentage, we made a comparison between the results obtained by our approach, based on fuzzy logic (dynamic adaptation of the amplitude of the emitted signal), with those obtained by transmitting with fixed amplitude, that is, 82 , 111, and 123, respectively, in DEC format. The correspondent Vpp values are described in Table 1. As depicted in Figure 8, the proposed approach allows obtaining better performance because the fuzzy controller dynamically determines the amplitude of the emitted signal, considering real transmission needs according to link quality and the master-slave distance.

When we transmit with low amplitude (82 in DEC format) we measured a lower TH/WL (80\%). This is due to the link attenuation that increases with the square of the distance. Most of lost packets are those ones that are sent to/from slaves farther away $(80 \mathrm{~m}$ and $100 \mathrm{~m})$. By transmitting with high amplitude (123 in DEC format) we obtained a worse result (86\%) than the transmission with average amplitude (111 in DEC format) due to the analog to digital converter saturation that occurs when the transmission involves closer devices (Slave 1 or Slave 2). The result obtained through the use of the fuzzy controller (99\%) is due to the dynamic adaptation of the amplitude of the emitted signal. The controller, in fact, dynamically responds to disturbances introduced into the network considering, at the same time, the master-slave distances.

The goodness of the proposed approach, skilled to deal with the problem of cross-coupling interference, has been tested by increasing the number of devices (up to 50) in a real three-phase scenario with 3 masters, one for each phase. The devices have been distributed in this way:

(i) Slaves S1, S4, S7, S10, S13, S16, S19, S22, S25, S28, S31, S34, S37, S40, S43, S46, and S49 were placed on Phase $\mathrm{R}$;

(ii) Slaves S2, S5, S8, S11, S14, S17, S20, S23, S26, S29, S32, S35, S38, S41, S44, S47, and S50 were placed on Phase $S$;

(iii) Slaves S3, S6, S9, S12, S15, S18, S21, S24, S27, S30, S33, S36, S39, S42, S45, and S48 were placed on Phase T.

Each phase is completely independent, with exception for the neutral conductor that joins the whole three-phase system. Due to the cross-coupling interference and the neutral wire, each signal transmitted by the Master R over the Phase R can be found on the other two phases with a certain phase shift and with lower amplitude. This disturbance can represent a serious problem; it can generate conflicts and unexpected answers over the network. According to Table 1, the signal transmitted by the Master R is an FSK modulated signal with amplitude:

(i) Vpp $=8.56 \mathrm{~V}$, if the Fuzzy Logic Controller output is 123 ;

(ii) $\mathrm{Vpp}=7.53 \mathrm{~V}$ if the FLC output is 111;

(iii) $\mathrm{Vpp}=5.82 \mathrm{~V}$ if the FLC output is 82 .

Several measures were carried out.

In Table 4, the Vpp values transmitted by Master $\mathrm{R}$ and the Vpp amplitude of the signal received by each slave, located on the three different phases, are shown. As it is possible to see, to transmit with low amplitude determines a reduction of cross-coupling interference and, therefore, a smaller number of conflicts and unexpected responses on the network and a higher TH/WL.

In Figure 9 it is possible to see how, in a network with 50 devices, the TH/WL obtained through the fuzzy controller is $94 \%$ while we measured $72 \%, 80 \%$, and $77 \%$ by transmitting with minimum, medium, and maximum amplitude, respectively, by adjusting the transmission power according to the distance and link quality.

Also in this case we carried out 2000 transmissions with 5,10 , and 50 devices, respectively, by transmitting the signal at fixed or variable amplitude according to the fuzzy logic. 
TABLE 4: Cross-coupling interference effect.

(a)

\begin{tabular}{lccccccccc}
\hline Master R & $\begin{array}{c}\text { S1 } \\
(\text { Phase R) }\end{array}$ & $\begin{array}{c}\text { S2 } \\
(\text { Phase })\end{array}$ & $\begin{array}{c}\text { S3 } \\
(\text { Phase T) }\end{array}$ & $\begin{array}{c}\text { S10 } \\
(\text { Phase R) }\end{array}$ & $\begin{array}{c}\text { S11 } \\
(\text { Phase S) }\end{array}$ & $\begin{array}{c}\text { S12 } \\
(\text { Phase T) }\end{array}$ & $\begin{array}{c}\text { S19 } \\
(\text { Phase R) }\end{array}$ & $\begin{array}{c}\text { S20 } \\
(\text { Phase S) }\end{array}$ & $\begin{array}{c}\text { S21 } \\
(\text { Phase T) }\end{array}$ \\
\hline Vpp = 8.56 V & 8.51 & 6.52 & 6.48 & 8.31 & 6.12 & 5.95 & 7.85 & 5.45 & 5.23 \\
Vpp = 7.53V & 7.49 & 5.54 & 5.47 & 7.27 & 5.15 & 5.02 & 6.79 & 4.35 & 4.29 \\
Vpp = 5.82 V & 5.63 & 3.28 & 3.41 & 5.46 & 3.15 & 3.08 & 4.98 & 2.78 & 2.63 \\
\hline
\end{tabular}

(b)

\begin{tabular}{lcccccc}
\hline Master R & $\begin{array}{c}\text { S31 } \\
(\text { Phase R) }\end{array}$ & $\begin{array}{c}\text { S32 } \\
(\text { Phase S) }\end{array}$ & $\begin{array}{c}\text { S33 } \\
(\text { Phase T) }\end{array}$ & $\begin{array}{c}\text { S46 } \\
(\text { Phase R) }\end{array}$ & $\begin{array}{c}\text { S47 } \\
(\text { Phase S) }\end{array}$ & $\begin{array}{c}\text { S48 } \\
(\text { Phase T) }\end{array}$ \\
\hline Vpp $=$ 8.56 V & 7.22 & 4.82 & 4.67 & 6.87 & 4.38 & 4.25 \\
Vpp $=7.53 \mathrm{~V}$ & 6.13 & 3.58 & 3.27 & 5.78 & 2.97 & 2.91 \\
Vpp $=5.82 \mathrm{~V}$ & 4.43 & 1.98 & 1.83 & 4.23 & 1.25 & 1.18 \\
\hline
\end{tabular}

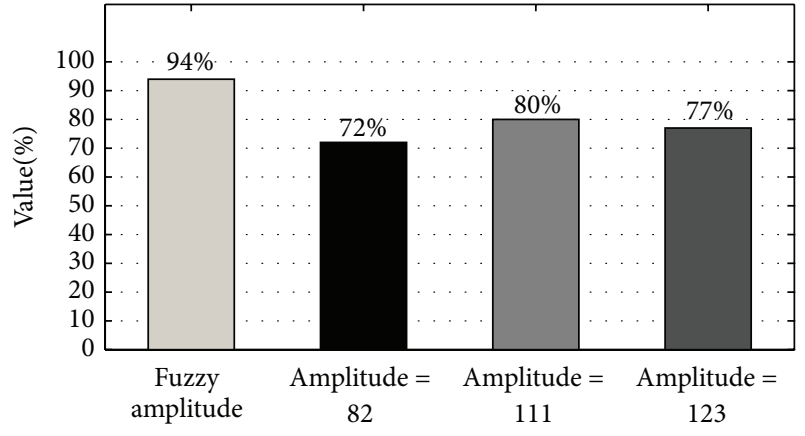

Figure 9: Throughput/Workload percentage comparison with 50 devices.

The results, depicted in Figure 10, demonstrate how the fuzzy controller ensures considerable savings, mainly when the number of devices, connected to the power grid, increases. The use of the fuzzy controller, in a network with 50 devices, determines a reduction of the power consumption of $37.8 \%$ and $24.3 \%$ compared to the case in which the transmission amplitude is maximum and medium, respectively. By transmitting with maximum amplitude, in fact, the average power consumption measured is $5.1 \mathrm{~W}$ while the average power consumption due to transmissions with medium amplitude is $4.6 \mathrm{~W}$. The use of the fuzzy controller allows reducing the average consumption up to $3.7 \mathrm{~W}$.

Finally in Figure 11 a typical transmitted signal on the main grid, FSK modulated, in shown. The measurements were carried out at the output of the isolation transformer. The left side of Figure 11, identified with the letter A, shows our user command "AT*WRFLU001998700000001" sent from the master to the Slave S1. It coincides with the implementation of its first output to duty cycle equal to $70 \%$.

The structure of a typical data packet is shown in Table 5.

(i) Command name: $\mathrm{AT}^{*}$ WRFLU.

(ii) Recipient identifier: 001 (first slave ID).

(iii) Sender identifier: 998 (ID master controller).

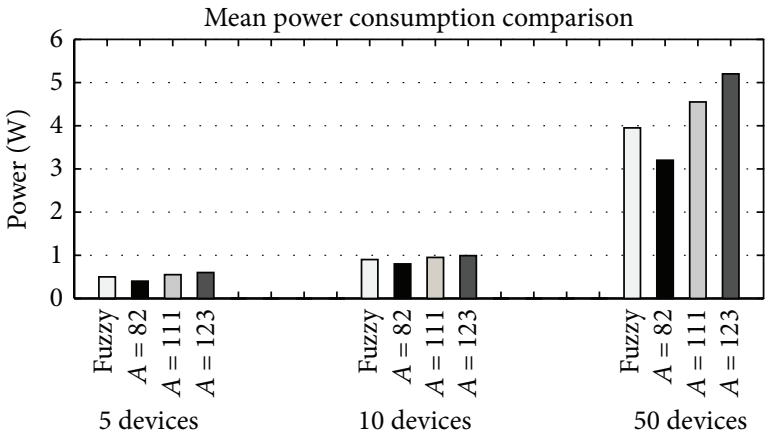

FIGURE 10: Power consumption comparison with 50 devices.

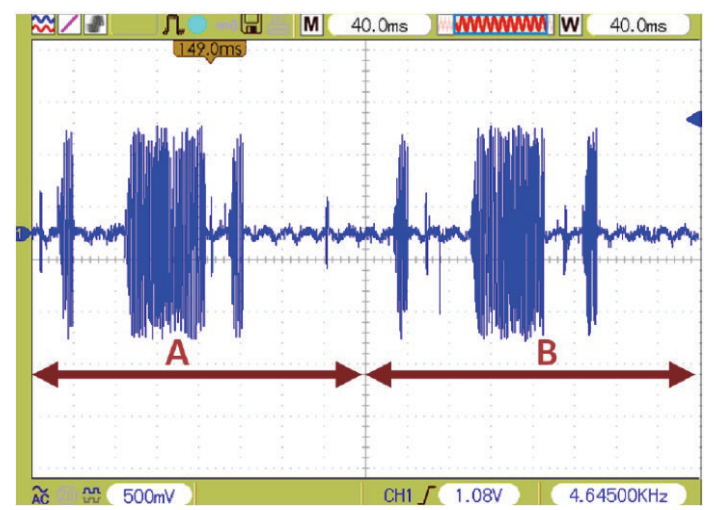

FIGURE 11: Master request slave response FSK data transmission.

(iv) Output duty cycle to implement: percentage for 4 channels available. In particular, it will contain 4 parameters, one for each channel [70]-[00]-[00]-[00] (70\% output channel $1,0 \%$ other outputs).

(v) Write flash command: it specifies whether or not to write the received command in flash (1 means true and 0 means false). 
TABLE 5: Typical transmitted frame.

\begin{tabular}{lcccc}
\hline $\begin{array}{l}\text { Command } \\
\text { name }\end{array}$ & $\begin{array}{c}\text { ID } \\
\text { receiver }\end{array}$ & $\begin{array}{c}\text { ID } \\
\text { sender }\end{array}$ & $\begin{array}{c}\text { Flux to } \\
\text { implement }\end{array}$ & $\begin{array}{l}\text { Write flash } \\
\text { command }\end{array}$ \\
\hline
\end{tabular}

\section{Conclusions}

In recent years, power line communications (PLCs) have been used in several fields and therefore several researchers have investigated different aspects, in order to optimize the use and the performance depending on the specific environment. In this work, we developed a fuzzy logic controller, into the System on Chip ADD1010, with the main aim to dynamically manage the amplitude with which each device transmits. The fuzzy controller, at first developed in MATLAB/Simulink, was subsequently inserted into the master device (C-source code generated through the embedded coder integrated in MATLAB). The obtained results are very promising. The TH/WL calculated through the proposed approach $(99 \%$ in the first scenario and $94 \%$ in the second scenario with 50 devices) demonstrate that almost all of the packets sent have been successfully received by their destinations, despite the problems of cross-coupling interference on a three-phase system. Even in terms of power consumption, the results are convincing. On average, the controller determines a lower consumption than the standard transmission with both maximum and average amplitude. As reported in the previous section, the fuzzy controller works better by increasing the number of network devices. In the case of 50 devices, in fact, the average power consumption reduction is $37.8 \%$ with respect to transmissions with maximum amplitude and $24.3 \%$ with respect to transmissions with medium amplitude. Clearly, if we consider transmissions with minimum amplitude, the power consumption produced by the approach here proposed is higher. This result is justified by a more effectively and efficiently management of the packets sent by the devices, thanks to the use of the fuzzy controller implemented in the master controller.

\section{Conflict of Interests}

The authors declare that there is no conflict of interests regarding the publication of this paper.

\section{References}

[1] IEEE Standard for Low-Frequency (less than $500 \mathrm{kHz}$ ) Narrowband Power Line Communications for Smart Grid Applications, pp. 1-269, 2013.

[2] C. T. Malangu, T. J. O. Afullo, and N. M. Ajumba, "Attenuation model for indoor multipath broadband PLC channels," in Proceedings of the IEEE-APS Topical Conference on Antennas and Propagation in Wireless Communications (APWC '12), pp. 1084-1087, Cape Town, South Africa, September 2012.

[3] P. A. C. Lopes, J. M. M. Pinto, and J. B. Gerald, "Dealing with unknown impedance and impulsive noise in the powerline communications channel," IEEE Transactions on Power Delivery, vol. 28, no. 1, pp. 58-66, 2013.
[4] R. Jahn, D. Lemmens, W. Foubert, and S. Uytterhoeven, "PLC Noise and impedance measurements on loads and in the distribution grid," in Proceedings of the 22nd International Conference and Exhibition on Electricity Distribution (CIRED '13), pp. 1-4, June 2013.

[5] G. Ren, S. Qiao, H. Zhao, C. Li, and Y. Hei, "Mitigation of periodic impulsive noise in OFDM-based power-line communications," IEEE Transactions on Power Delivery, vol. 28, no. 2, pp. 825-834, 2013.

[6] C. Gabriel, H. Horia, and O. A. Pop, "Developing powerline carrier applications on embedded systems," in Proceedings of the 27th International Spring Seminar on Electronics Technology: Meeting the Challenges of Electronics Technology Progress, vol. 1, pp. 158-161, 2004.

[7] D. Guezgouz, D. E. Chariag, Y. Raingeaud, and J.-C. Le Bunetel, "Modeling of electromagnetic interference and PLC transmission for loads shedding in a microgrid," IEEE Transactions on Power Electronics, vol. 26, no. 3, pp. 747-754, 2011.

[8] M. Collotta, S. Tirrito, and R. Caponetto, "Flexible street lamp lighting system management through power line," in Proceedings of the International Symposium on Power Electronics, Electrical Drives, Automation and Motion (SPEEDAM '14), pp. 1021-1026, 2014.

[9] M. O. Ahmed and L. Lampe, "Power line communications for low-voltage power grid tomography," IEEE Transactions on Communications, vol. 61, no. 12, pp. 5163-5175, 2013.

[10] M. Korki, C. Zhang, and H. L. Vu, "Performance evaluation of PRIME in smart grid," in Proceedings of the IEEE International Conference on Smart Grid Communications (SmartGridComm '13), pp. 294-299, IEEE, Vancouver, BC, Canada, October 2013.

[11] P. Alliance, PRIME Alliance, http://www.prime-alliance.org/.

[12] Y. Kim, J. N. Bae, and J. Y. Kim, "Performance of power line communication systems with noise reduction scheme for smart grid applications," IEEE Transactions on Consumer Electronics, vol. 57, no. 1, pp. 46-52, 2011.

[13] M. Korki, N. Hosseinzadeh, H. L. Vu, T. Moazzeni, and C. H. Foh, "Impulsive noise reduction of a narrowband power line communication using optimal nonlinearity technique," in Proceedings of the Australasian Telecommunication Networks And Applications Conference (ATNAC '11), pp. 1-4, Melbourne, Australia, November 2011.

[14] P. Torio and M. G. Sanchez, "Method to cancel impulsive noise from power-line communication systems by processing the information in the idle carriers," IEEE Transactions on Power Delivery, vol. 27, no. 4, pp. 2421-2422, 2012.

[15] M. Nassar, A. Dabak, I. H. Kim, T. Pande, and B. L. Evans, "Cyclostationary noise modeling in narrowband powerline communication for Smart Grid applications," in Proceedings of the IEEE International Conference on Acoustics, Speech, and Signal Processing (ICASSP '12), pp. 3089-3092, Kyoto, Japan, March 2012.

[16] G. Bumiller, L. Lampe, and H. Hrasnica, "Power line communication networks for large-scale control and automation systems," IEEE Communications Magazine, vol. 48, no. 4, pp. 106-113, 2010.

[17] A. Usman and S. H. Shami, "Evolution of communication technologies for smart grid applications," Renewable and Sustainable Energy Reviews, vol. 19, pp. 191-199, 2013.

[18] ADD1010 datasheet, http://www.mymectronic.com/datasheet/. 11015_ADD1010DK.pdf.

[19] L. A. Zadeh, “Toward extended fuzzy logic: a first step," Fuzzy Sets and Systems, vol. 160, no. 21, pp. 3175-3181, 2009. 
[20] M. Mokhtar, F. Piltan, M. Mirshekari, A. Khalilian, and O. Avatefipour, "Design minimum rule-base fuzzy inference nonlinear controller for second order nonlinear system," International Journal of Intelligent Systems and Applications, vol. 7, pp. 79-88, 2014.

[21] A. Hedi, B. O. Slim, and B. S. Slim, "Hardware implementation of fuzzy logic controller," in Proceedings of the 12th IEEE International Conference on Electronics, Circuits and Systems (ICECS '05), pp. 1-4, Gammarth, Tunisia, December 2005.

[22] R. Antony and J. Ilow, "An adaptive threshold strategy for soft decision viterbi decoding," in Proceedings of the Canadian Conference on Electrical and Computer Engineering: Toward a Caring and Humane Technology (CCECE '03), pp. 1659-1662, May 2003.

[23] N. Mogharreban and L. F. Dilalla, "Comparison of defuzzification techniques for analysis of non-interval data," in Annual Meeting of the North American Fuzzy Information Processing Society (NAFIPS '06), pp. 257-260, 2006. 

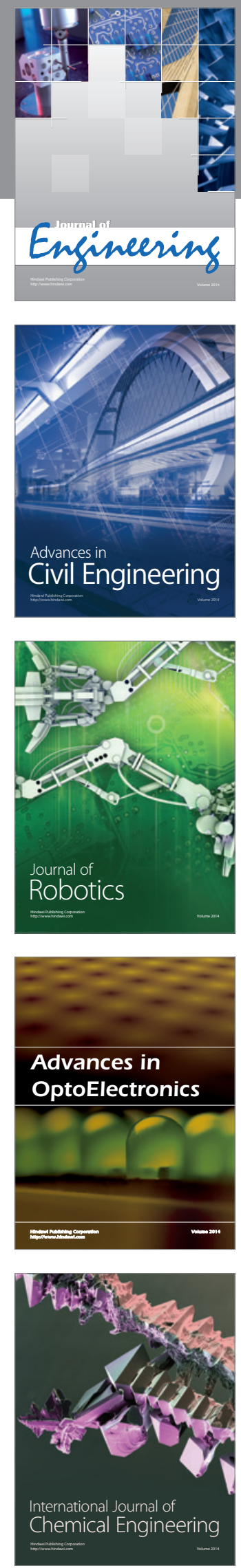

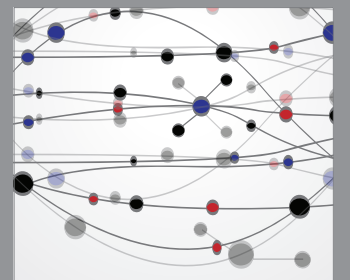

The Scientific World Journal
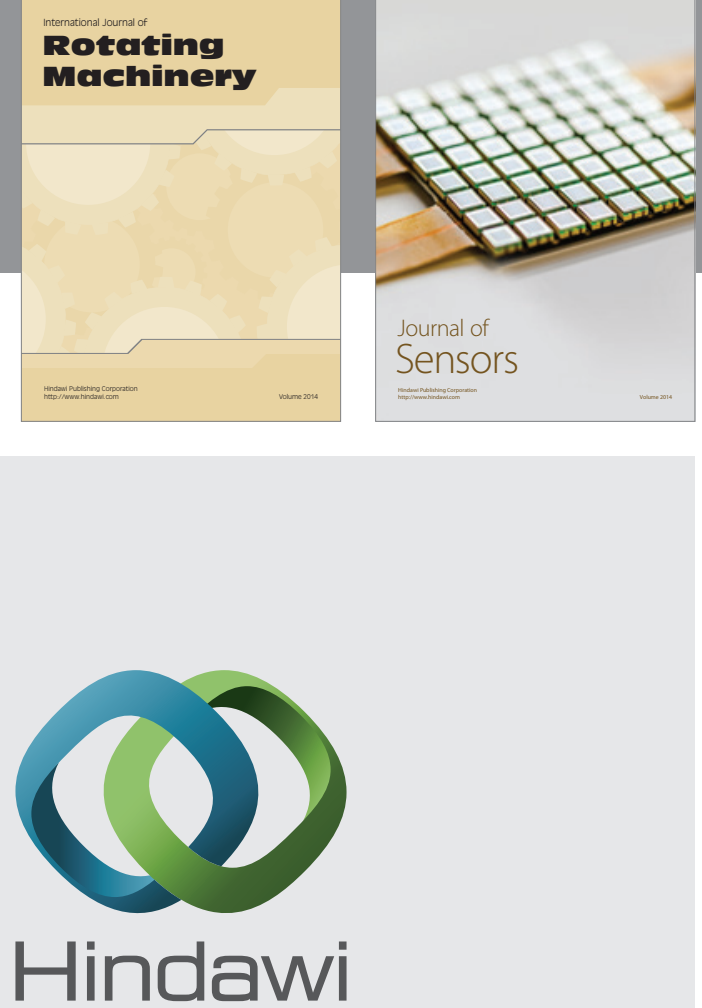

Submit your manuscripts at http://www.hindawi.com
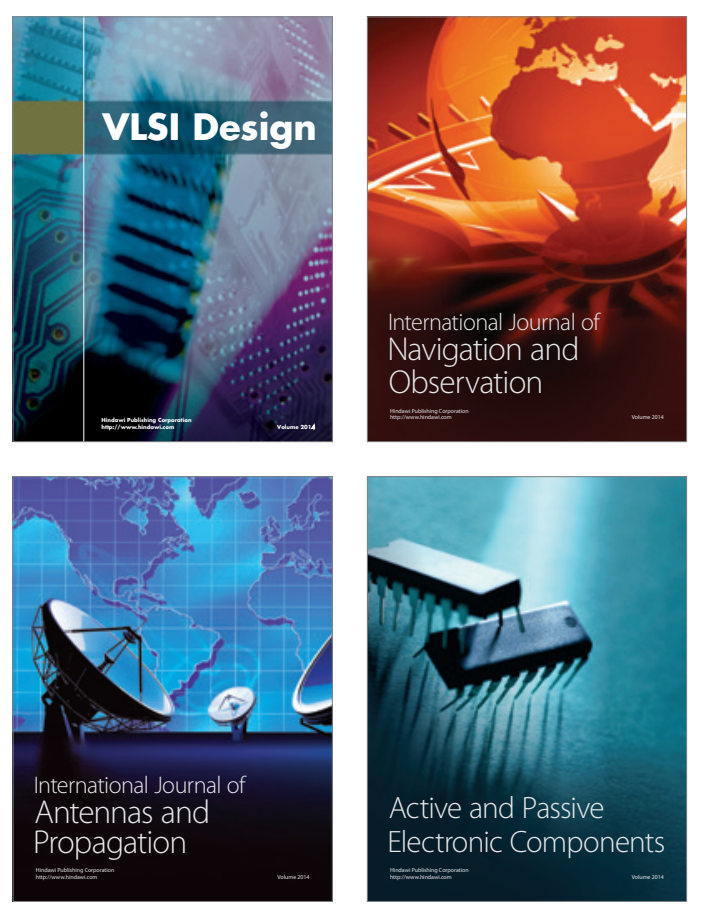
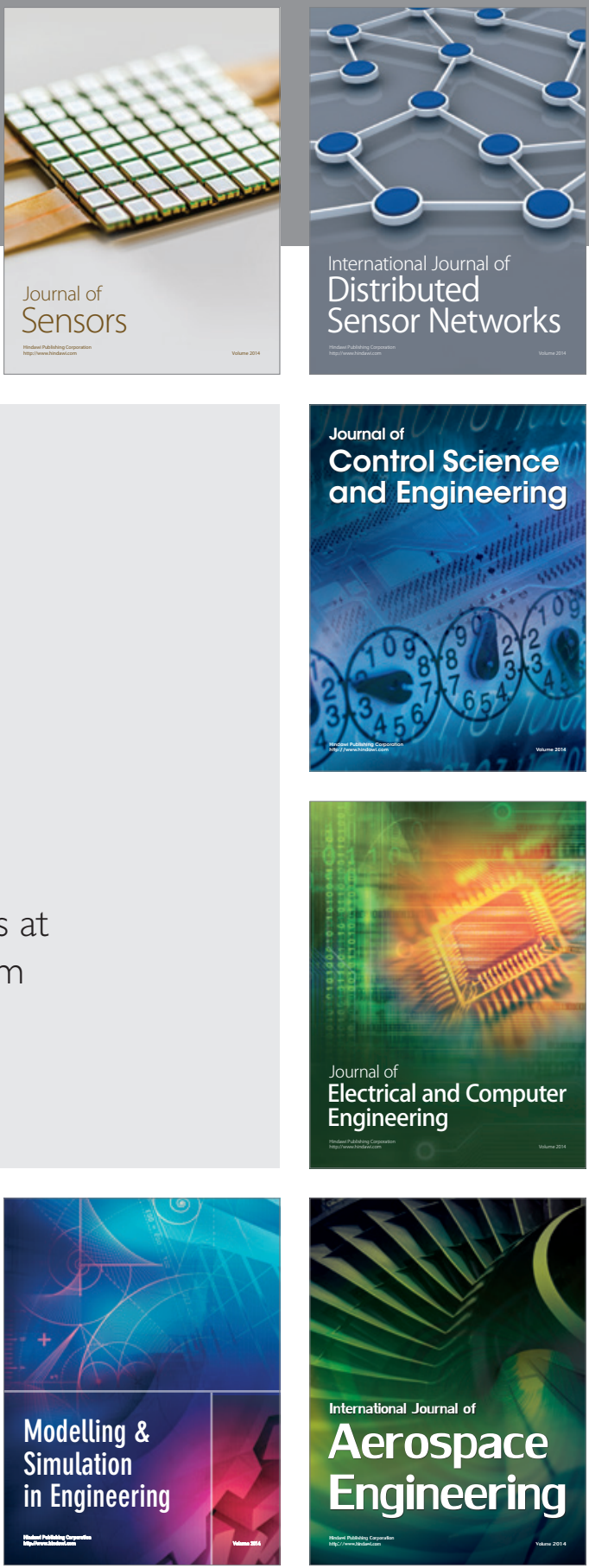

Journal of

Control Science

and Engineering
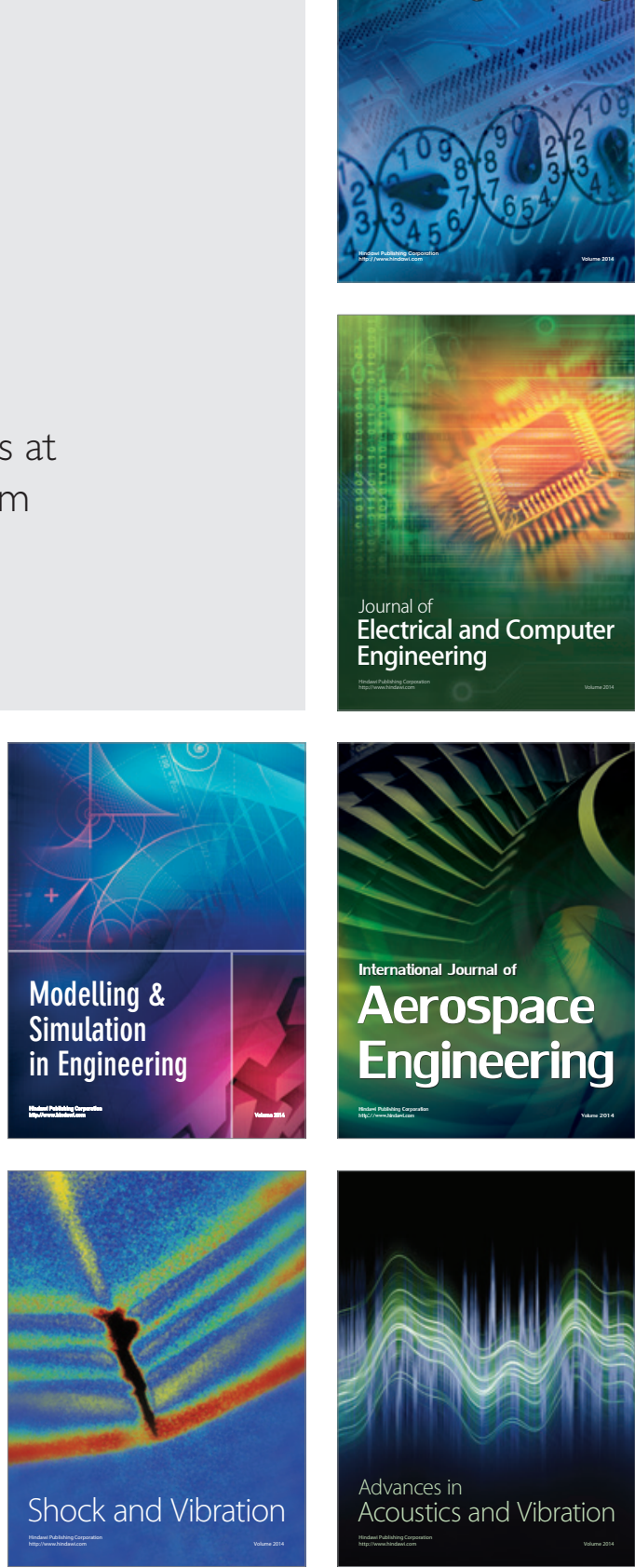